\title{
APPLICATIONS OF MEASURE OF NONCOMPACTNESS TO COUPLED FIXED POINTS AND SYSTEMS OF INTEGRAL EQUATIONS
}

\author{
A. SAMADI \\ Received 18 February, 2018
}

\begin{abstract}
In this paper, some existence theorems involving generalized contractive conditions with respect to a measure are proved. By applying our results, we study some coupled fixed point theorems, and discuss the existence of solutions for a class of the system of integral equations. Finally, an example is included to show the efficiency of our results.
\end{abstract}

2010 Mathematics Subject Classification: 47H08; 47H10

Keywords: measure of noncompactness, coupled point theorem, integral equations

\section{INTRODUCTION}

Integral equations are used naturally in applied problems, such as in a lot of problems in physics and engineering. Also, especially integral equations have been linked in many applications in the kinetic theory of gases, the theory of radioactive transfer, see for example $[9,11,12]$. The existence theorems for nonlinear integral equations have been studied in many papers with the help of the technique of measures of noncompactness which was initiated by Kuratowski [10]. The Kuratowski measure of noncompactness has attracted the interest of mathematicians working in the study of functional equations, ordinary and partial differential equations and many other fields. If fact, since measures of noncompactness are functions suitable for measuring the degree of noncompactness of a given set, they are very useful tools in the wide area of functional analysis such as the metric fixed point theory and the theory of operator equations in Banach spaces (see [3,13,14]). In this paper, first we recall some essential concepts and results that will be used later. Then, we give some new fixed point theorems applying the technique of measure of noncompactness. In the third section, we apply our results to a coupled fixed point. Finally in order to indicate the applicability of our results, we study the problem of the existence of solutions for a class of system of integral equations.

The author was supported by Department of Mathematics, Miyaneh Branch, Islamic Azad University, Miyaneh, Iran. 
Now we recall some notations, definitions and theorems which will be needed further on.

Throughout this paper we assume that $E$ is a real Banach space with norm $\|$.$\| and$ zero element $\theta$. Let $X$ be a nonempty subset of $E$. The closure and the closed convex hull of $X$ will be denoted by $\bar{X}$ and Convc(X), respectively. Moreover, let us denote by $M_{E}$ the family of all nonempty and bounded subsets of $E$ and by $N_{E}$ its subfamily consisting of all relatively compact sets.

The following definition of measure of noncompactness will be used in our results.

Definition 1 ([6]). A mapping $\mu: M_{E} \longrightarrow[0, \infty)$ is called a measure of noncompactness if it satisfies the following conditions:

(1) The family $\operatorname{Ker} \mu=\left\{X \in M_{E}: \mu(X)=0\right\}$ is nonempty and $\operatorname{Ker} \mu \subseteq N_{E}$.

(2) $X \subseteq Y \Longrightarrow \mu(X) \leq \mu(Y)$.

(3) $\mu(\bar{X})=\mu(X)$.

(4) $\mu(\operatorname{Conv}(X))=\mu(X)$.

(5) $\mu(\lambda X+(1-\lambda) Y) \leq \lambda \mu(X)+(1-\lambda) \mu(Y)$ for $\lambda \in[0,1]$.

(6) If $\left\{X_{n}\right\}$ is a sequence of closed sets from $M_{E}$ such that $X_{n+1} \subseteq X_{n}$ for $n=1,2, \ldots$ and $\lim _{n \rightarrow \infty} \mu\left(X_{n}\right)=0$, then $\bigcap_{n=1}^{\infty} X_{n}$ is nonempty.

Theorem 1 (Schauder [3]). Let $U$ be a nonempty, bounded, closed and convex subset of a Banach space $E$. Then every continuous and compact map $F: U \longrightarrow U$ has at leat one fixed point in $U$.

Theorem 2 (Darbo[8]). Let $Q$ be a nonempty, closed, bounded and convex subset of a Banach space $E$ and $F: Q \longrightarrow Q$ be a continuous mapping. Assume that there exists a constant $k \in[0,1)$ such that $\mu(F X) \leq k \mu(X)$ for any nonempty subset $X$ of $Q$. Then $F$ has a fixed point in $Q$.

The following definitions, theorems and examples will be used further on.

Definition 2 ([7]). An element $(x, y) \in X \times X$ is called coupled fixed point of the mapping $F: X \times X \longrightarrow X$ if $F(x, y)=x$ and $F(y, x)=y$.

Theorem 3 ([6]). Suppose $\mu_{1}, \mu_{2}, \ldots, \mu_{n}$ are measures of noncompactness in $E_{1}, E_{2}, \ldots, E_{n}$ respectively. Moreover assume that the function $F:[0, \infty)^{n} \longrightarrow$ $[0, \infty)$ is convex and

$F\left(x_{1}, x_{2}, \ldots, x_{n}\right)=0$ if and only if $x_{i}=0$ for $i=1,2, \ldots, n$. Then

$$
\mu(X)=F\left(\mu_{1}\left(X_{1}\right), \mu_{2}\left(X_{2}\right), \ldots, \mu_{n}\left(X_{n}\right)\right)
$$

defines a measure of noncompactness in $E_{1} \times E_{2} \times \cdots \times E_{n}$ where $X_{i}$ denote the natural projection of $X$ into $E_{i}$ for $i=1,2, \ldots, n$.

Example 1 ([2]). Let $\mu$ be a measure of noncompactness in the Banach space $E$ and $F(x, y)=x+y$ for $(x, y) \in[0, \infty)^{2}$. Then $F$ has all the properties in Theorem 3. Hence $\bar{\mu}(X)=\mu\left(X_{1}\right)+\mu\left(X_{2}\right)$ is a measure of noncompactness in the space $E \times E$ where $X_{i}, i=1,2$ denote the natural projections of $X$ into $E$. 
Now, inspired by Definition 2.4 of [1], the following definition is introduced which is basic for our main results.

Definition 3. Let $\Theta$ denote the class of those functions $\phi: \mathbb{R}_{+}^{2} \rightarrow \mathbb{R}_{+}$which satisfy the following conditions

$\left(\delta_{1}\right) \phi\left(t_{1}, t_{2}\right)=\phi$ is increasing in $t_{1}$ and $t_{2}$.

$\left(\delta_{2}\right) t_{n+1}<\phi\left(t_{n}, t_{n}\right)$ implies that $t_{n+1}<t_{n}$ for each positive sequence $\left\{t_{n}\right\}$.

$\left(\delta_{3}\right) \phi(u, u) \leq u$ for each $u \in[0, \infty)$.

Example 2. Let $\phi: \mathbb{R}_{+}^{2} \rightarrow \mathbb{R}_{+}$defined by

$$
\phi\left(t_{1}, t_{2}\right)=a t_{1}+b t_{2}
$$

where $a+b=1$ and $b \neq 1$. Then $\phi \in \Theta$

Definition 4 ([4]). Let $F:(0, \infty) \rightarrow R$ and $\theta:(0, \infty) \rightarrow(0, \infty)$ be two mappings. Throughout the paper, let $\Delta$ be the set of all pairs $(\theta, F)$ satisfying the following:

$\left(\Delta_{1}\right) \theta\left(t_{n}\right) \nrightarrow 0$ for each strictly decreasing sequence $\left\{t_{n}\right\}$;

$\left(\Delta_{2}\right) \mathrm{F}$ is strictly increasing function;

$\left(\Delta_{3}\right)$ for each sequence $\left\{\alpha_{n}\right\}$ of positive numbers, $\lim _{n \rightarrow \infty} \alpha_{n}=0$ if and only if $\lim _{n \rightarrow \infty} F\left(\alpha_{n}\right)=-\infty$

$\left(\Delta_{4}\right)$ If $\left\{t_{n}\right\}$ be a decreasing sequence such that $t_{n} \rightarrow 0$ and $\theta\left(t_{n}\right)<F\left(t_{n}\right)-F\left(t_{n+1}\right)$, then we have $\sum_{n=1}^{\infty} t_{n}<\infty$.

Example 3 ([4]). Let $F(t)=\ln (t)$ and $\theta(t)=-\ln (\alpha(t))$ for each $t \in(0, \infty)$, where $\alpha:(0, \infty) \rightarrow(0,1)$ satisfies $\lim \sup \alpha(s)<1$, for all $t \in(0, \infty)$. Then $(\theta, F) \in \Delta$.

\section{SOME FIXED POINT RESULTS VIA A NEW GENERALIZED CONTRACTIVE CONDITION}

Now inspired by the existing contractive condition in [1], the main result of this paper is stated.

Theorem 4. Let $C$ be a nonempty bounded, closed and convex subset of a Banach space $E$. Assume $T: C \longrightarrow C$ is a continuous operator satisfying

$$
\theta(\psi(\mu(T(X))))+f(\psi(\mu(T(X)))) \leq f(\phi(\psi(\mu(X)), \psi(\mu((X)))))
$$

for all nonempty subset $X$ of $C$, where $\mu$ is an arbitrary measure of noncompactness defined in $E, \psi:[0, \infty) \longrightarrow[0, \infty)$ is nondecreasing such that $\psi(t)=0$ if and only if $t=0, \phi \in \Theta$ and $(\theta, f) \in \Delta$. Then $T$ has a fixed point in $C$.

Proof. Define a sequence $\left\{C_{n}\right\}$ by letting $C_{0}=C$ and $C_{n}=\operatorname{Conv}\left(T C_{n-1}\right), n \geq$ 1. If there exists an integer $N \geq 0$ such that $\mu\left(C_{N}\right)=0$, then $C_{N}$ is relatively compact and Theorem 1 implies that $T$ has a fixed point. So we assume that $\mu\left(C_{n}\right)>0$ for each $n \in N$. By our assumptions, we get 


$$
\begin{aligned}
\theta\left(\psi\left(\mu\left(C_{n_{+}}\right)\right)\right)+f\left(\psi\left(\mu\left(C_{n+1}\right)\right)\right) & \leq f\left(\phi\left(\psi\left(\mu\left(C_{n}\right)\right), \psi\left(\mu\left(C_{n}\right)\right)\right)\right) \\
& \leq f\left(\psi\left(\mu\left(C_{n}\right)\right)\right) .
\end{aligned}
$$

Consequently, by $\left(\Delta_{2}\right)$, we have

$$
\psi\left(\mu\left(C_{n+1}\right)\right)<\psi\left(\mu\left(C_{n}\right)\right) .
$$

Since the sequence $\left\{\psi\left(\mu\left(C_{n}\right)\right)\right\}$ is non-increasing sequence, there exists $t \geq 0$ such that $\lim _{n \rightarrow \infty} \psi\left(\mu\left(C_{n}\right)\right)=t$. Now we show that $t=0$. On the contrary, assume that $t>0$. From (2.2) we have

$$
\Sigma_{i=1}^{\infty} \theta\left(\psi\left(\mu\left(C_{i+2}\right)\right)\right) \leq f\left(\psi\left(\mu\left(C_{2}\right)\right)\right)-f\left(\psi\left(\mu\left(C_{n+1}\right)\right)\right) .
$$

Keeping in mind our assumptions we have $\theta\left(\psi\left(\mu\left(C_{n}\right)\right)\right) \nrightarrow 0$. So, we infer that $\Sigma_{i=1}^{i=n-1} \theta\left(\psi\left(\mu\left(C_{i+2}\right)\right)\right)=\infty$ and consequently $\lim _{n \rightarrow \infty} f\left(\psi\left(\mu\left(C_{n+1}\right)\right)\right)=-\infty$. So, by $\Delta_{3}$ we get $\psi\left(\mu\left(C_{n}\right)\right) \rightarrow 0$ which is a contradiction. Hence, $\psi\left(\mu\left(C_{n}\right)\right) \longrightarrow 0$ as $n \longrightarrow \infty$. Now we prove that $\mu\left(C_{n}\right) \rightarrow 0$. Since $\left\{\psi\left(\mu\left(C_{n}\right)\right)\right\}$ is a decreasing sequence and $\psi$ is nondecreasing, we obtain that $\left\{\mu\left(C_{n}\right)\right\}$ is a decreasing sequence of positive numbers. Consequently there exists $r \geq 0$ such that $\lim _{n \rightarrow \infty} \mu\left(C_{n}\right)=r^{+}$. Since $\psi$ is nondecreasing, we arrive that

$$
\psi(r) \leq \psi\left(\mu\left(C_{n}\right)\right) .
$$

Letting $n \longrightarrow \infty$ in (2.4), we have $\psi(r) \leq 0$. So $r=0$ which implies that $\mu\left(C_{n}\right) \rightarrow$ 0 . On the other hand, since $C_{n+1} \subseteq C_{n}$ and $\mu\left(C_{n}\right) \rightarrow 0$, from condition (6) of Definition 1 we obtain that $C_{\infty}=\cap_{n=1}^{\infty} C_{n}$ is nonempty, closed, convex and $C_{\infty} \subseteq C$. Moreover, taking in to account our assumptions we infer that $C_{\infty}$ is invariant under the operator $T$ and $C_{\infty} \in \operatorname{Ker} \mu$. Consequently, from Theorem 1 we deduce that $T$ has a fixed point.

Corollary 1. Let $C$ be a nonempty bounded, closed and convex subset of the Banach space $E$. Assume $T: C \longrightarrow C$ is a continuous operator satisfying

$$
\theta(\mu(T(X)))+f(\mu(T(X))) \leq f(\mu(X))
$$

for all nonempty subset $X$ of $C$, where $\mu$ is an arbitrary measure of noncompactness defined in $E$ and $(\theta, f) \in \Delta$. Then $T$ has at least one fixed point in $C$.

Proof. Obviously, (2.5) is a special case of (2.1) with $\psi(t)=t$ and $\phi\left(t_{1}, t_{2}\right)=t_{1}$. Hence, the application of Theorem 4 completes the proof.

Corollary 2. Let $C$ be a nonempty bounded, closed and convex subset of a Banach space E. Assume $T: C \longrightarrow C$ is a continuous operator satisfying

$$
\mu(T(X)) \leq \alpha(\mu(T(X))) \mu(X)
$$


for all nonempty subset $X$ of $C$, where $\mu$ is an arbitrary measure of noncompactness defined in $E$ and $\alpha:(0, \infty) \longrightarrow[0,1)$ with $\lim \sup \alpha(t)<1$ for all $r \geq 0$. Then $T$ has a fixed point in $C$.$$
t \rightarrow r^{+}
$$

Proof. By applying Corollary 1 with $f(t)=\ln (t)$ and $\theta(t)=\ln (\alpha(t))$, the proof will be completed.

Corollary 3. Let $C$ be a nonempty bounded, closed and convex subset of the Banach space $E$. Assume $T: C \longrightarrow C$ is a continuous operator satisfying

$$
\mu(T(X)) \leq \varphi(\mu(T(X))) \mu(X)
$$

for all nonempty subset $X$ of $C$, where $\mu$ is an arbitrary measure of noncompactness defined in $E$ and $\varphi:(0, \infty) \longrightarrow[0,1)$ is a non-decreasing function. Then $T$ has a fixed point in $C$.

Proof. Since $\varphi$ is non-decreasing function, we have $\lim \sup \varphi(t)<1$ for all $r \geq 0$. Thus, Corollary 2 completes the proof.

$$
t \rightarrow r^{+}
$$

Theorem 5. Let $C$ be a nonempty bounded, closed and convex subset of a Banach space E. Assume $T: C \longrightarrow C$ is a continuous operator satisfying

$$
\theta(\mu(X))+f(\mu(T(X))) \leq f(\mu(X))
$$

for all nonempty subset $X$ of $C$, where $\mu$ is an arbitrary measure of noncompactness defined in $E$ and $(\theta, f) \in \Delta$. Then $T$ has a fixed point in $C$.

Proof. Similar to the proof of Theorem 4, we can construct the sequence $\left\{C_{n}\right\}$ such that

$$
\theta\left(\mu\left(C_{n}\right)\right)+f\left(\mu\left(C_{n+1}\right)\right) \leq f\left(\mu\left(C_{n}\right)\right),
$$

which yields that $\mu\left(C_{n+1}\right)<\mu\left(C_{n}\right)$. So there exists $r \geq 0$ such that $\mu\left(C_{n}\right) \rightarrow r$. On the other hand, from (2.9) we have

$$
\Sigma_{i=1}^{i=n-1} \theta\left(\psi\left(\mu\left(C_{i+1}\right)\right)\right)<f\left(\mu\left(C_{2}\right)\right)-f\left(\mu\left(C_{n+1}\right) .\right.
$$

Now by using the technique in Theorem 4 , we have $\mu\left(C_{n}\right) \rightarrow 0$. Therefore, taking into account that $C_{n+1} \subseteq C_{n}$, from condition (6) of Definition 1 we conclude that $C_{\infty}=\cap_{n=1}^{\infty} C_{n}$ is nonempty, closed, convex and $C_{\infty} \subseteq C$. Moreover, the set $C_{\infty}$ is invariant under the operator $T$ and belong to $\operatorname{Ker} \mu$. Consequently, from Theorem 1 we deduce that $T$ has a fixed point.

Corollary 4. Let $C$ be a nonempty bounded, closed and convex subset of the Banach space E. Assume $T: C \longrightarrow C$ is a continuous operator satisfying

$$
\mu(T(X))) \leq \alpha(\mu(X)) \mu(X)
$$


for all nonempty subset $X$ of $C$, where $\mu$ is an arbitrary measure of noncompactness defined in $E$ and $\alpha:(0, \infty) \longrightarrow[0,1)$ with $\lim \sup \alpha(t)<1$ for all $r \geq 0$. Then $T$ has a fixed point in $C$. $t \rightarrow r^{+}$

Proof. Taking $f(t)=\ln (t)$ and $\theta(t)=\ln (\alpha(t))$ in Theorem 5, the result follows.

Corollary 5. Let $C$ be a nonempty bounded, closed and convex subset of the Banach space E. Assume $T: C \longrightarrow C$ is a continuous operator satisfying

$$
\mu(T(X))) \leq \varphi(\mu(X)) \mu(X)
$$

for all nonempty subset $X$ of $C$, where $\mu$ is an arbitrary measure of noncompactness defined in $E$ and $\varphi:[0, \infty) \longrightarrow[0,1)$ is a non-decreasing function. Then $T$ has a fixed point in $C$.

Proof. Since $\varphi$ is non-decreasing, $\operatorname{so} \lim \sup \varphi(t)<1$ for all $r \geq 0$. Consequently,

$$
t \rightarrow r^{+}
$$

applying Corollary 4 with $\varphi=\alpha$, we have the result.

\section{COUPLED FiXED POINT RESUltS}

In this section, as an application of Theorem 4 we study the existence of coupled fixed point to a special class of operators. Let $\Psi$ denote all functions $\psi:[0, \infty) \rightarrow$ $[0, \infty)$ such that

(1) $\psi$ is nondecreasing and $\psi(t)=0$ if and only if $t=0$,

(2) $\psi(t+s) \leq \psi(t)+\psi(s)$ for all $t, s \geq 0$.

Theorem 6. Let $C$ be a nonempty bounded, closed and convex subset of the Banach space E. Assume $T: C \times C \longrightarrow C$ is a continuous operator satisfying

$$
\begin{aligned}
\theta\left(\psi\left(\mu\left(T\left(X_{1} \times X_{2}\right)\right)\right)\right) & +f\left(\psi\left(\mu\left(T\left(X_{1} \times X_{2}\right)\right)\right)\right) \\
& \leq \frac{1}{2} f\left(\phi\left(\psi\left(\mu\left(X_{1}\right)+\mu\left(X_{2}\right)\right), \psi\left(\mu\left(X_{1}\right)+\mu\left(X_{2}\right)\right)\right)\right)
\end{aligned}
$$

for all nonempty subsets $X_{1}, X_{2} \subset C$, where $\mu$ is an arbitrary measure of noncompactness defined in $E, \psi \in \Psi, \phi \in \Theta$ and $(\theta, f) \in \Delta$ such that

$$
\theta(t+s) \leq \theta(t)+\theta(s) \quad \text { and } \quad f(t+s) \leq f(t)+f(s) .
$$

Then $T$ has at least a coupled fixed point.

Proof. We consider a mapping $\bar{T}: C \times C \rightarrow C \times C$ defined by $\bar{T}(x, y)=(T(x, y), T(y, x))$. Since $T$ is continuous, the continuity of $\bar{T}$ is followed. From example1, we know that $\bar{\mu}(X)=\mu\left(X_{1}\right)+\mu\left(X_{2}\right)$ defines a measure of noncompactness on $E \times E$ for any $X_{1}, X_{2} \subseteq C$ where $X_{i}=1,2$ indicate the natural projection of $X$ into $E$. Let 
$X \subseteq C \times C$ be a nonempty subset. Then, due to (3.1) and condition (2) of Definition 1 we infer that

$$
\begin{aligned}
\theta(\psi(\bar{\mu}(\bar{T}(X)))) & +f(\psi(\bar{\mu}(\bar{T}(X)))) \\
& \leq \theta\left(\psi\left(\bar{\mu}\left(T\left(X_{1} \times X_{2}\right) \times T\left(X_{2} \times X_{1}\right)\right)\right)\right) \\
& +f\left(\psi\left(\bar{\mu}\left(T\left(X_{1} \times X_{2}\right) \times T\left(X_{2} \times X_{1}\right)\right)\right)\right) \\
& =\theta\left(\psi\left(\mu\left(T\left(X_{1} \times X_{2}\right)\right)+\mu\left(T\left(X_{2} \times X_{1}\right)\right)\right)\right. \\
& +f\left(\psi\left(\mu\left(T\left(X_{1} \times X_{2}\right)\right)+\mu\left(T\left(X_{2} \times X_{1}\right)\right)\right)\right) \\
& \leq \theta\left(\psi\left(\mu\left(T\left(X_{1} \times X_{2}\right)\right)\right)\right)+\theta\left(\psi\left(\mu\left(T\left(X_{2} \times X_{1}\right)\right)\right)\right) \\
& +f\left(\psi\left(\mu\left(T\left(X_{1} \times X_{2}\right)\right)\right)\right)+f\left(\psi\left(\mu\left(T\left(X_{2} \times X_{1}\right)\right)\right)\right) \\
& \leq f\left(\phi\left(\psi\left(\mu\left(X_{1}\right)+\mu\left(X_{2}\right)\right), \psi\left(\mu\left(X_{1}\right)+\mu\left(X_{2}\right)\right)\right)\right) \\
& =f(\phi(\psi(\bar{\mu}(X)), \psi(\bar{\mu}(X)))) .
\end{aligned}
$$

So all the conditions of Theorem 4 hold true and $\bar{T}$ has a fixed point. Hence, $\mathrm{T}$ has a coupled fixed point.

Corollary 6. Let $C$ be a nonempty bounded, closed and convex subset of a Banach space $E$. Assume $T: C \times C \longrightarrow C$ is a continuous operator satisfying

$$
\theta\left(\mu\left(T\left(X_{1} \times X_{2}\right)\right)\right)+f\left(\mu\left(T\left(X_{1} \times X_{2}\right)\right)\right) \leq f\left(\mu\left(X_{1}\right)+\mu\left(X_{2}\right)\right)
$$

for all nonempty subsets $X_{1}, X_{2} \subset C$, where $\mu$ is an arbitrary measure of noncompactness defined in $E$ and $(\theta, f) \in \Delta$ such that $\theta(t+s) \leq \theta(t)+\theta(s)$ and $f(t+s) \leq f(t)+f(s)$. Then $T$ has at least a coupled fixed point.

Proof. Take $\phi\left(t_{1}, t_{2}\right)=t_{1}$ and $\psi=I$ in Theorem 6.

Corollary 7. Let $C$ be a nonempty bounded, closed and convex subset of the Banach space $E$. Assume $T: C \times C \longrightarrow C$ is a continuous operator satisfying

$$
\mu\left(T\left(X_{1} \times X_{2}\right)\right) \leq \alpha\left(\mu\left(T\left(X_{1} \times X_{2}\right)\right)\right)\left(\mu\left(X_{1}\right)+\mu\left(X_{2}\right)\right)
$$

for all nonempty subsets $X_{1}, X_{2} \subset C$, where $\mu$ is an arbitrary measure of noncompactness defined in $E$ and $\alpha:(0, \infty) \longrightarrow[0,1)$ with limsup $\alpha(t)<1$ for all $r \geq 0$. Then $T$ has at least a coupled fixed point.

Proof. Let $\theta(t)=\ln (\alpha(t))$ and $f(t)=\ln (t)$. So from (3.4) we have

$$
\theta\left(\mu\left(T\left(X_{1} \times X_{2}\right)\right)\right)+f\left(\mu\left(T\left(X_{1} \times X_{2}\right)\right)\right) \leq f\left(\mu\left(X_{1}\right)+\mu\left(X_{2}\right)\right)
$$

for all nonempty subsets $X_{1}, X_{2} \subseteq C$. Now, Corollary 6 guarantees that $\mathrm{T}$ has a coupled fixed pont. 


\section{SOLVABILITY OF SYSTEMS OF INTEGRAL EQUATIONS}

This section is devoted to the study of the existence of solutions for the systems of integral equations

$$
\begin{aligned}
x(t) & =F(t, h(t, x(\alpha(t)), y(\alpha(t))), \\
& (T x))(t) \int_{0}^{\eta(t)} \varphi(t, s) g(t, s, x(\gamma(s)), y(\gamma(s)) d s) \\
y(t) & =F(t, h(t, y(\alpha(t)), x(\alpha(t))), \\
& (T y))(t) \int_{0}^{\eta(t)} \varphi(t, s) g(t, s, y(\gamma(s)), x(\gamma(s)) d s),
\end{aligned}
$$

in the space $B C\left(R_{+}\right) \times B C\left(R_{+}\right)$consisting of all bounded and continuous real functions on $R_{+}$. For $x \in B C\left(R_{+}\right)$the norm of $x$ is defined by $\|x\|=\sup \{|x(t)|$ : $t \geq 0\}$. Now, we reacal the definition of measure of noncompactness in the space $B C\left(R_{+}\right)$which was introduced by Banas in [5]. Fix a nonempty bounded subset $X$ of $B C\left(R_{+}\right)$and a positive number $K>0$. For $x \in X$ and $\varepsilon>0$ put

$$
\begin{aligned}
\omega^{K}(x, \varepsilon) & =\sup \{|x(t)-y(t)| ; t, s \in[0, K],|t-s| \leq \varepsilon\}, \\
\omega^{K}(X, \varepsilon) & =\sup \left\{\omega^{K}(x, \varepsilon) ; x \in X\right\}, \\
\omega_{0}^{K}(X) & =\lim _{\varepsilon \rightarrow 0} \omega^{K}(X, \varepsilon), \\
\omega_{0}(X) & =\lim _{K \rightarrow \infty} \omega_{0}^{K}(X) .
\end{aligned}
$$

Furthermore, for a fixed number $t \in R^{+}$, let us define the following equation:

$$
\begin{aligned}
X(t) & =\{x(t) ; x \in X\}, \\
\operatorname{diam} X(t) & =\sup \{|x(t)-y(t)| ; x, y \in X\} .
\end{aligned}
$$

Finally, let

$$
\mu(X)=\omega_{0}(X)+\limsup _{t \rightarrow \infty} \operatorname{diam} X(t) .
$$

Banas [5] proved that the above function is a measure of noncompactness in the space $B C\left(R_{+}\right)$. Now, the existence of solutions for the integral equations (4.1) is studied under the following assumptions.

(1) $\gamma, \alpha, \eta: R_{+} \rightarrow R_{+}$are continuous functions and $\alpha(t) \rightarrow \infty$ as $t \rightarrow \infty$.

(2) The functions $F: R_{+} \times R \times R \rightarrow R$ and $h: R_{+} \times R \times R \rightarrow R$ are continuous functions and there exist positive real number $\tau>0$ such that

$$
\begin{gathered}
\left|F\left(t, x_{1}, x_{2}\right)-F\left(t, y_{1}, y_{2}\right)\right| \leq e^{-\tau}\left(\left|x_{1}-y_{1}\right|+\left|x_{2}-y_{2}\right|\right), \\
\left|h\left(t, x_{1}, x_{2}\right)-h\left(t, y_{1}, y_{2}\right)\right| \leq e^{-\tau}\left(\left|x_{1}-y_{1}\right|+\left|x_{2}-y_{2}\right|\right),
\end{gathered}
$$

for $t \in R_{+}$and $x_{1}, x_{2}, y_{1}, y_{2} \in R$. 
(3) The functions $t \rightarrow F(t, 0,0)$ and $t \rightarrow h(t, 0,0)$ are bounded on $R_{+}$i.e $M_{1}, M_{2}<$ $\infty$ where $M_{1}=\sup \{F(t, 0,0) ; t \geq 0\}, M_{2}=\sup \{h(t, 0,0) ; t \geq 0\}$.

(4) $T: B C\left(R_{+}\right) \rightarrow B C\left(R_{+}\right)$is a continuous operator such that

$$
\left|(T x)\left(t_{1}\right)-(T x)\left(t_{2}\right)\right| \leq \psi_{1}\left(\left|x\left(t_{2}\right)-x\left(t_{1}\right)\right|\right),|(T x)(t)| \leq a+b\|x\|,
$$

$$
|(T x)(t)-(T u)(t)| \leq \psi_{1}(|x(t)-u(t)|) .
$$

for $x \in B C\left(R_{+}\right)$and $t_{2}, t_{1} \in R_{+}$, where $\psi_{1}: R_{+} \longrightarrow R_{+}$is continuous and nondecreasing with $\psi_{1}(0)=0$ and $a, b$ are positive real numbers.

(5) $\varphi: \mathbb{R}^{+} \times \mathbb{R}^{+} \longrightarrow \mathbb{R}^{+}$is continuous on $\mathbb{R}^{+} \times \mathbb{R}^{+}$and the function $t \longrightarrow \varphi(t, s)$ is nondecreasing for each $s \in \mathbb{R}^{+}$.

(6) There exist continuous functions

$$
a, b: R_{+} \rightarrow R_{+} \quad g: \mathbb{R}^{+} \times \mathbb{R}^{+} \times \mathbb{R} \times \mathbb{R} \longrightarrow \mathbb{R}
$$

such that

$$
\lim _{t \rightarrow \infty} a(t) \int_{0}^{\eta(t)} b(s) d s=0 \quad|\varphi(t, s) g(t, s, x, y)| \leq a(t) b(s),
$$

for all $t, s \in R^{+}$and $x, y \in R$ such that $s \leq t$.

(7) There exists a positive solution $r_{0}$ of the inequality

$$
2 r e^{-2 \tau}+M_{2} e^{-\tau}+e^{-\tau}(a+b r) q+M_{1} \leq r
$$

where $q=\sup \left\{a(t) \int_{0}^{\eta(t)} b(s) d s ; t \geq 0\right\}$.

Theorem 7. Under assumptions (1) - (7), Eq (4.1) has at least one solution in the space $B C\left(R_{+}\right) \times B C\left(R_{+}\right)$.

Proof. Let us consider the operator $G$ on the space $B C\left(R_{+}\right) \times B C\left(R_{+}\right)$by

$$
\begin{aligned}
G(x, y)(t) & =F(t, h(t, x(\alpha(t)), y(\alpha(t))), \\
& (T x)(t) \int_{0}^{\eta(t)} \varphi(t, s) g(t, s, x(\gamma(s)), y(\gamma(s)) d s) .
\end{aligned}
$$

We know that the space $B C\left(R_{+}\right) \times B C\left(R_{+}\right)$is equipped with the norm

$$
\|(x, y)\|=\|x\|_{\infty}+\|y\|_{\infty} .
$$


On the other hand, obviously $G$ is continuous. Taking into account our assumptions, we infer that

$$
\begin{aligned}
|G(x, y)(t)| & \leq e^{-\tau}(|h(t, x(\alpha(t)), y(\alpha(t)))-h(t, 0,0)| \\
& \left.+|h(t, 0,0)|+\left|(T x)(t) \int_{0}^{\eta(t)} \varphi(t, s) u(t, s, x(\gamma(s)), y(\gamma(s))) d s\right|\right) \\
& +|F(t, 0,0,0,0)| \leq e^{-\tau}\left(e^{-\tau}(|x(\alpha(t))|+|y(\alpha(t))|)\right. \\
& \left.+M_{2}+(a+b\|x\|) a(t) \int_{0}^{\eta(t)} b(s) d s\right)+M_{1} \\
& \leq e^{-2 \tau}(\|x\|+\|y\|)+e^{-\tau} M_{2}+e^{-\tau}(a+b\|x\|) q+M_{1} .
\end{aligned}
$$

Consequently, from (4.5) and condition (7) we infer that $G\left(\overline{B_{r_{0}}} \times \overline{B_{r_{0}}}\right) \subseteq \overline{(4.5)}$. Now, we indicate that $G$ is a continuous on $\overline{B_{r_{0}}} \times \overline{B_{r_{0}}}$. To do this, let $\varepsilon>0$ be an arbit-

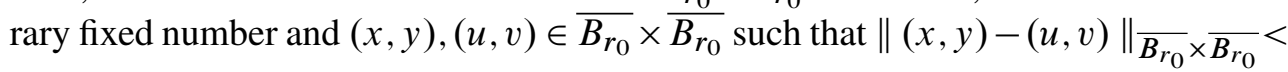
$\frac{\varepsilon}{2}$. Then, we have

$$
\begin{aligned}
& |G(x, y)(t)-G(u, v)(t)| \\
& \leq e^{-\tau}(|h(t, x(\alpha(t)), y(\alpha(t)))-h(t, u(\alpha(t)), v(\alpha(t)))|) \\
& +e^{-\tau}\left(\mid(T x)(t) \int_{0}^{\eta(t)} \varphi(t, s) u(t, s, x(\gamma(s)), y(\gamma(s))) d s\right. \\
& \left.-(T u)(t) \int_{0}^{\eta(t)} \varphi(t, s) g(t, s, u(\gamma(s)), v(\gamma(s)))\right) d s \mid \\
& \leq e^{-2 \tau}(|x(\alpha(t))-u(\alpha(t))|+|y(\alpha(t))-v(\alpha(t))|) \\
& +e^{-\tau}|(T x)(t)| \mid \int_{0}^{\eta(t)} \varphi(t, s)(g(t, s, x(\gamma(s)), y(\gamma(s))) \\
& -g(t, s, u(\gamma(s)), v(\gamma(s)))) d s \mid \\
& +e^{-\tau}|(T x)(t)-(T u)(t)| \times \\
& \left|\int_{0}^{\eta(t)} \varphi(t, s) g(t, s, u(\gamma(s)), v(\gamma(s))) d s\right| .
\end{aligned}
$$


Thus, from (4.6) we have

$$
\begin{aligned}
& |G(x, y)(t)-G(u, v)(t)| \\
& \leq e^{-2 \tau}(\|x-u\|+\|y-v\|) \\
& +e^{-\tau}(a+b\|x\|) \mid \int_{0}^{\eta(t)} \varphi(t, s)(g(t, s, x(\gamma(s)), y(\gamma(s))) \\
& -g(t, s, u(\gamma(s)), v(\gamma(s)))) d s \mid \\
& +e^{-\tau}\|T x-T u\| a(t) \int_{0}^{\eta(t)} b(s) d s .
\end{aligned}
$$

Furthermore, by Condition (6) there exists $T>0$ such that

$$
a(t) \int_{0}^{\eta(t)} b(s) d s<\frac{\varepsilon}{2} .
$$

for all $t>T$.

Hence, by combining the inequalities (4.7) and (4.8), we deduce that

$$
\begin{aligned}
|G(x, y)(t)-G(u, v)(t)| & \leq e^{-2 \tau} \varepsilon+2 e^{-\tau}(a+b\|x\|) \varepsilon \\
& +\|(T x)-(T u)\| e^{-\tau} \varepsilon .
\end{aligned}
$$

Now we define the equality $\omega^{T}(g, \varepsilon)$ as follows:

$$
\begin{gathered}
\omega^{T}(g, \varepsilon)=\sup \left\{|g(t, s, x, y)-g(t, s, u, v)|: t \in[0, T], s \in\left[0, \eta_{T}\right] ; x, y, u\right. \\
, v \in\left[-r_{0}, r_{0}\right],\|(x, y)-(u, v)\|_{\left.B C\left(R_{+}\right) \times B C\left(R_{+}\right)<\varepsilon\right\},}
\end{gathered}
$$

where

$$
\eta_{T}=\sup \{\eta(t) ; t \in[0, T]\} .
$$

On the other hand from (4.6) for an arbitrary fixed $t \in[0, T]$ we have

$$
\begin{aligned}
|G(x, y)(t)-G(u, v)(t)| & \leq e^{-2 \tau}(\|x-u\|+\|y-v\|) \\
& +e^{-\tau}(a+b\|x\|)\left|\int_{0}^{\eta(t)} \varphi(t, s) \omega^{T}(g, \varepsilon) d s\right| \\
& +e^{-\tau}\|x-u\| a(t) \int_{0}^{\eta(t)} b(s) d s .
\end{aligned}
$$

By applying the continuity of $g$ on $[0, T] \times\left[0, \eta_{T}\right] \times\left[-r_{0}, r_{0}\right] \times\left[-r_{0}, r_{0}\right]$, we have $\omega^{T}(g, \varepsilon) \rightarrow 0$ as $\varepsilon \rightarrow 0$. Hence, due to (4.9) and (4.10) we conclude that $G$ is continuous. Now, let $T, \varepsilon \in R_{+}$and $X_{1}, X_{2}$ are arbitrary nonempty subsets of $\overline{B_{r_{0}}}$. Assume $t_{1}, t_{2} \in[0, T]$ such that $\left|t_{2}-t_{1}\right| \leq \varepsilon$ and $\eta\left(t_{1}\right) \leq \eta\left(t_{2}\right)$. In view of our assumptions, 
for $(x, y) \in X_{1} \times X_{2}$ we get

$$
\begin{aligned}
& \left|G(x, y)\left(t_{2}\right)-G(x, y)\left(t_{1}\right)\right| \\
& \leq \mid G\left(t_{2}, h\left(t_{2}, x\left(\alpha\left(t_{2}\right)\right), y\left(\alpha\left(t_{2}\right)\right),\right.\right. \\
& \left.(T x)\left(t_{2}\right) \int_{0}^{\eta\left(t_{2}\right)} \varphi\left(t_{2}, s\right) g\left(t_{2}, s, x(\gamma(s)), y(\gamma(s))\right) d s\right) \\
& -G\left(t_{1}, h\left(t_{1}, x\left(\alpha\left(t_{1}\right)\right), y\left(\alpha\left(t_{1}\right)\right),(T x)\left(t_{1}\right) \times\right.\right. \\
& \left.\int_{0}^{\eta\left(t_{1}\right)} \varphi\left(t_{1}, s\right) g\left(t_{1}, s, x(\gamma(s)), y(\gamma(s))\right) d s\right) \mid \\
& \leq e^{-\tau}\left(\left|h\left(t_{2}, x\left(\alpha\left(t_{2}\right)\right), y\left(\alpha\left(t_{2}\right)\right)\right)-h\left(t_{2}, x\left(\alpha\left(t_{1}\right)\right), y\left(\alpha\left(t_{1}\right)\right)\right)\right|\right. \\
& +\mid h\left(t_{2}, x\left(\alpha\left(t_{1}\right)\right), y\left(\alpha\left(t_{1}\right)\right)-h\left(t_{1}, x\left(\alpha\left(t_{1}\right)\right), y\left(\alpha\left(t_{1}\right)\right)\right) \mid\right. \\
& +e^{-\tau} \mid(T x)\left(t_{2}\right) \int_{0}^{\eta\left(t_{2}\right)} \varphi\left(t_{2}, s\right) g\left(t_{2}, s, x(\gamma(s)), y(\gamma(s))\right) d s \\
& \left.-(T x)\left(t_{1}\right) \int_{0}^{\eta\left(t_{1}\right)} \varphi\left(t_{1}, s\right) g\left(t_{1}, s, x(\gamma(s)), y(\gamma(s))\right) d s \mid\right) .
\end{aligned}
$$

Thus, from (4.11) we get

$$
\begin{aligned}
& \left|G(x, y)\left(t_{2}\right)-G(x, y)\left(t_{1}\right)\right| \\
& \leq e^{-2 \tau}\left(\left|x\left(\alpha\left(t_{2}\right)\right)-x\left(\alpha\left(t_{1}\right)\right)\right|+\left|y\left(\alpha\left(t_{2}\right)\right)-y\left(\alpha\left(t_{1}\right)\right)\right|\right)+e^{-\tau} \omega_{r_{0}}^{T}(h, \varepsilon) \\
& +e^{-\tau}\left(\mid(T x)\left(t_{2}\right) \int_{0}^{\eta\left(t_{2}\right)} \varphi\left(t_{2}, s\right) g\left(t_{2}, s, x(\gamma(s)), y(\gamma(s))\right) d s\right. \\
& \left.-(T x)\left(t_{1}\right) \int_{0}^{\eta\left(t_{1}\right)} \varphi\left(t_{1}, s\right) g\left(t_{1}, s, x(\gamma(s)), y(\gamma(s))\right) d s \mid\right) \\
& \leq e^{-2 \tau}\left(\omega^{T}\left(x, \omega^{T}(\alpha, \varepsilon)\right)+\omega^{T}\left(y, \omega^{T}(\alpha, \varepsilon)\right)\right)+e^{-\tau} \omega_{r_{0}}^{T}(h, \varepsilon) \\
& +e^{-\tau}\left(\mid(T x)\left(t_{2}\right) \int_{0}^{\eta\left(t_{2}\right)} \varphi\left(t_{2}, s\right) g\left(t_{2}, s, x(\gamma(s)), y(\gamma(s))\right) d s\right. \\
& \left.-(T x)\left(t_{1}\right) \int_{0}^{\eta\left(t_{1}\right)} \varphi\left(t_{1}, s\right) g\left(t_{1}, s, x(\gamma(s)), y(\gamma(s))\right) d s \mid\right) .
\end{aligned}
$$


On the other hand, we have

$$
\begin{aligned}
& \mid(T x)\left(t_{2}\right) \int_{0}^{\gamma\left(t_{2}\right)} \varphi\left(t_{2}, s\right) g\left(t_{2}, s, x(\gamma(s)), y(\gamma(s))\right) d s \\
& -(T x)\left(t_{1}\right) \int_{0}^{\eta\left(t_{1}\right)} \varphi\left(t_{1}, s\right) g\left(t_{1}, s, x(\gamma(s)), y(\gamma(s))\right) d s \mid \\
& \leq \mid(T x)\left(t_{2}\right) \int_{0}^{\eta\left(t_{2}\right)} \varphi\left(t_{2}, s\right) g\left(t_{2}, s, x(\gamma(s)), y(\gamma(s))\right) d s \\
& -(T x)\left(t_{1}\right) \int_{0}^{\eta\left(t_{2}\right)} \varphi\left(t_{2}, s\right) g\left(t_{2}, s, x(\gamma(s)), y(\gamma(s))\right) d s \mid \\
& +\mid(T x)\left(t_{1}\right) \int_{0}^{\eta\left(t_{2}\right)} \varphi\left(t_{2}, s\right) g\left(t_{2}, s, x(\gamma(s)), y(\gamma(s))\right) d s \\
& +(T x)\left(t_{1}\right) \int_{0}^{\eta\left(t_{2}\right)} \varphi\left(t_{1}, s\right) g\left(t_{2}, s, x(\gamma(s)), y(\gamma(s))\right) d s \mid \\
& +\mid(T x)\left(t_{1}\right) \int_{0}^{\eta\left(t_{2}\right)} \varphi\left(t_{1}, s\right) g\left(t_{2}, s, x(\gamma(s)), y(\gamma(s))\right) d s \\
& +\left|(T x)\left(t_{1}\right)\left\|\int_{\eta\left(t_{1}\right)}^{\eta\left(t_{2}\right)} \mid \varphi\left(t_{1}, s\right)\right\| g\left(t_{2}, s, x(\gamma(s)), y(\gamma(s))\right)\right. \\
& +\left|(T x)\left(t_{1}\right) \int_{0}^{\eta\left(t_{1}\right)} \varphi\left(t_{1}, s\right) g\left(t_{1}, s, x(\gamma(s)), y(\gamma(s))\right) d s\right| \\
& \left.+\mid(T x)\left(t_{2}\right)-(T x)\left(t_{1}\right) \| \int_{0}^{\eta\left(t_{2}\right)} \varphi\left(t_{2}, s\right) g\left(t_{2}, s, x(\gamma(s)), y \gamma(s)\right)\right) d s \mid \\
& +
\end{aligned}
$$


Now, take into consideration (4.12) and (4.13) we have

$$
\begin{aligned}
\left|G(x, y)\left(t_{2}\right)-G(x, y)\left(t_{1}\right)\right| & \leq e^{-2 \tau}\left(\omega^{T}\left(x, \omega^{T}(\alpha, \varepsilon)\right)+\omega^{T}\left(y, \omega^{T}(\alpha, \varepsilon)\right)\right) \\
& +e^{-\tau} \omega_{r_{0}}^{T}(h, \varepsilon)+e^{-\tau}\left(\psi_{1}(\omega(x, \varepsilon)) \times\right. \\
& \left.\|\varphi\| a\left(t_{2}\right) \int_{0}^{\eta\left(t_{2}\right)} b(s) d s\right)+e^{-\tau}(a+b\|x\|) \times \\
& \omega_{\varphi}(\varepsilon, .) \times a\left(t_{2}\right) \int_{0}^{\eta\left(t_{2}\right)} b(s) d s \\
& +e^{-\tau}(a+b\|x\|)\|\varphi\| \times \\
& \omega_{r_{0}}^{T}(g, \varepsilon) \omega^{T}(\eta, \varepsilon) .
\end{aligned}
$$

where

$$
\begin{aligned}
& \omega_{r_{0}}^{T}(g, \varepsilon)=\sup \left\{\left|g\left(t_{1}, s, x, y\right)-g\left(t_{2}, s, x, y\right)\right|: t_{1}, t_{2} \in[0, T],\right. \\
& \left.\left|t_{1}-t_{2}\right|<\varepsilon, s \in\left[0, \eta_{T}\right], x, y \in\left[-r_{0}, r_{0}\right]\right\}, \\
& \omega^{T}\left(x, \omega^{T}(\alpha, \varepsilon)\right)=\sup \left\{\left|x\left(t_{1}\right)-x\left(t_{2}\right)\right|: t_{1}, t_{2} \in[0, T],\left|t_{1}-t_{2}\right| \leq \omega^{T}(\alpha, \varepsilon)\right\}, \\
& \omega_{r_{0}}^{T}(h, \varepsilon)=\sup \left\{\left|h\left(t_{2}, x, y\right)-h\left(t_{1}, x, y\right)\right|: t_{1}, t_{2} \in[0, T],\left|t_{1}-t_{2}\right| \leq \varepsilon\right. \\
& \left., x, y \in\left[-r_{0}, r_{0}\right]\right\}, \\
& \omega_{\varphi}(\epsilon, .)=\sup \{|\varphi(t, s)-\varphi(\dot{t}, s): t, \dot{t} \in[0, T],| t-\dot{t} \mid \leq \varepsilon\}, \\
& \omega^{T}(\alpha, \varepsilon)=\sup \left\{\left|\alpha\left(t_{2}\right)-\alpha\left(t_{1}\right)\right| ; t_{2}, t_{1} \in[0, T],\left|t_{2}-t_{1}\right|<\varepsilon\right\} .
\end{aligned}
$$

Moreover, in the light of the uniform continuity of the functions $g, h$ and $\varphi$ on $[0, T] \times\left[0, \eta_{T}\right] \times\left[-r_{0}, r_{0}\right] \times\left[-r_{0}, r_{0}\right],[0, T] \times\left[-r_{0}, r_{0}\right] \times\left[-r_{0}, r_{0}\right]$ and $[0, T] \times[0, T]$, we have $\omega_{r_{0}}^{T}(g, \varepsilon) \longrightarrow 0, \omega_{r_{0}}^{T}(h, \varepsilon) \rightarrow 0$ and $\omega_{\varphi}(\epsilon,.) \rightarrow 0$. Also because of the uniform continuity of $\alpha, \eta$ on $[0, T]$ we have $\omega^{T}(\alpha, \varepsilon) \rightarrow 0, \omega^{T}(\eta, \varepsilon) \rightarrow 0$. Now, this remarks and the inequalities in (4.14) imply that

$$
\omega_{0}^{T}\left(G\left(X_{1} \times X_{2}\right), \varepsilon\right) \leq e^{-2 \tau}\left(\omega_{0}^{T}\left(X_{1}\right)+\omega_{0}^{T}\left(X_{2}\right)\right),
$$

and hence

$$
\omega_{0}\left(G\left(X_{1} \times X_{2}\right)\right) \leq e^{-2 \tau}\left(\omega_{0}\left(X_{1}\right)+\omega_{0}\left(X_{2}\right)\right) .
$$


Now, arbitrary elements $(x, y),(u, v) \in X_{1} \times X_{2}$ are chosen so that for $t \in R^{+}$, we have

$$
\begin{aligned}
|G(x, y)(t)-G(u, v)(t)| & \leq e^{-\tau}(|x(\alpha(t))-u(\alpha(t))|+|y(\alpha(t))-v(\alpha(t))|) \\
& +e^{-\tau}\left(\mid(T x)(t) \int_{0}^{\eta(t)} \varphi(t, s) g(t, s, x(\gamma(s)), y(\gamma(t))) d s\right. \\
& \left.-(T u)(t) \mid \int_{0}^{\eta(t)} \varphi(t, s) g(t, s, u(\gamma(s)), v(\gamma(t))) d s\right) \mid \\
& \leq e^{-\tau}\left(\operatorname{diam} X_{1}(\alpha(t))+\operatorname{diam}_{2}(\alpha(t))\right) \\
& +e^{-\tau}((a+b\|x\|)+(c+d\|u\|)) a(t) \int_{0}^{\eta(t)} b(s) d s .
\end{aligned}
$$

Now, using (4.18) and the notion of diameter of a set, we have

$$
\begin{aligned}
\operatorname{diam} G\left(X_{1} \times X_{2}\right)(t) & \left.\leq e^{-\tau}\left(\operatorname{diam} X_{1}(\alpha(t))+\operatorname{diam} X_{2}(\alpha(t))\right)\right) \\
& +e^{-\tau}((a+b\|x\|)+(c+d\|x\|)) a(t) \int_{0}^{\eta(t)} b(s) d s,
\end{aligned}
$$

and hence

$$
\begin{aligned}
\limsup _{t \rightarrow \infty} \operatorname{diam} G\left(X_{1} \times X_{2}\right)(t) & \leq e^{-\tau}\left(\limsup _{t \rightarrow \infty} \operatorname{diam} X_{1}(\alpha(t))\right. \\
& \left.+\operatorname{limsupdiam}_{t \rightarrow \infty} X_{2}(\alpha(t))\right) .
\end{aligned}
$$

Combining (4.17), (4.20) and (4.2) we get

$$
\mu\left(G\left(X_{1} \times X_{2}\right)\right) \leq e^{-\tau}\left(\mu\left(X_{1}\right)+\mu\left(X_{2}\right)\right)
$$

By passing to logarithms, we earn

$$
\ln \left(\mu\left(G\left(X_{1} \times X_{2}\right)\right) \leq \ln \left(e^{-\tau}\left(\mu\left(X_{1}\right)+\mu\left(X_{2}\right)\right)\right) .\right.
$$

Consequently,

$$
\tau+\ln \left(\mu\left(G\left(X_{1} \times X_{2}\right)\right) \leq \ln \left(\mu\left(X_{1}\right)+\mu\left(X_{2}\right)\right)\right) .
$$

Then all conditions of Corollary 6 hold true with $F(t)=\ln (t)$ and $\theta(t)=\tau$ for all $t \in R_{+}$. Consequently, from Corollary $6 G$ has a coupled fixed point in the space $\left.B C\left(R_{+}\right) \times B C\left(R_{+}\right)\right)$.

Example 4. Now, we will study the following system of integral equations

$$
\left\{\begin{array}{l}
x(t)=e^{-t-\tau} \cos \left(\frac{e^{-t-\tau}}{(1+|x|+|y|)}+\cos \left(\frac{1}{1+|x(t)|}\right) \int_{0}^{t} e^{t} \arctan \left(\frac{e^{-3 t+s}}{8+|x|+|y|}\right) d s\right) \\
y(t)=e^{-t-\tau} \cos \left(\frac{e^{-t-\tau}}{(1+|x|+|y|)}+\cos \left(\frac{1}{1+|y(t)|}\right) \int_{0}^{t} e^{t} \arctan \left(\frac{e^{-3 t+s}}{8+|y|+|x|}\right) d s\right) .
\end{array}\right.
$$

This system is a special case of the system of integral equations (4.1) with 


$$
\begin{aligned}
F(t, x, y) & =e^{-t-\tau} \cos (x+y), \quad h(t, x, y)=\frac{e^{-t-\tau}}{1+|x|+|y|}, \\
(T x)(t) & \left.=\cos \left(\frac{1}{1+|x(t)|}\right), \quad g(t, s, x, y)=\arctan \left(\frac{e^{-3 t+s}}{8+|y|+|x|}\right)\right), \\
\varphi(t, s) & =e^{t}, \quad \alpha(t)=\eta(t)=\gamma(t)=t .
\end{aligned}
$$

It is easily seen that $\alpha, \eta, \gamma$ satisfy the assumption (1). Further, the function $F(t, 0,0)=$ $e^{-\tau-t}$ is bounded with $M_{1}=e^{-\tau}$. Also, the function $|h(t, 0,0)|=e^{-t-\tau}$ is bounded with $M_{2}=e^{-\tau}$. Since $F(t, x, y)=e^{-t-\tau} \cos (x+y)$ and $h(t, x, y)=\frac{e^{-t-\tau}}{1+|x|+|y|}$, then, for all $t \in R_{+}$and $x_{1}, x_{2}, y_{1}, y_{2} \in R$, we have

$$
\begin{gathered}
\left|F\left(t, x_{1}, y_{1}\right)-F\left(t, x_{2}, y_{2}\right)\right| \leq e^{-\tau}\left(\left|x_{1}-x_{2}\right|+\left|y_{1}-y_{2}\right|\right), \\
\left|h\left(t, x_{1}, y_{1}\right)-h\left(t, x_{2}, y_{2}\right)\right| \leq e^{-\tau}\left(\left|x_{1}-x_{2}\right|+\left|y_{1}-y_{2}\right|\right) .
\end{gathered}
$$

Consequently, $F$ and $h$ satisfy the assumption (2). In this example $(T x)(t)=\cos \left(\frac{1}{1+|x(t)|}\right)$ verifies assumption (4) with $a=1, b=0$ and $\psi_{1}=t$. Moreover, assumption (5) holds with $\varphi(t, s)=e^{t}$. On the other hand, for all $t, s \in R_{+}$ and $x, y \in R$ with $s \leq t$, we get

$$
|\varphi(t, s) g(t, s, x, y)| \leq e^{-2 t+s} .
$$

Thus, assumption (6) holds with $a(t)=e^{-2 t}$ and $b(s)=e^{s}$. Consequently, the existent inequality in assumption (7) has the form

$$
2 r e^{-\tau}+e^{-2 \tau}+e^{-\tau} \leq r .
$$

It is easily seen that the last inequality have a positive solution. Consequently, all the conditions of Theorem 7 are satisfied and Theorem 7 guarantees that the system of integral equation (4.22) has at least on solution in the space $B C\left(R_{+}\right) \times B C\left(R_{+}\right)$.

\section{REFERENCES}

[1] H. Baghani and M. Ramezani, "A fixed point theorem for a new class of set-valued mappings in r-complete(not nesessarily complete) metric spaces.” Filomat, vol. 31, no. 12, pp. 3875-3884, 2017, doi: 10.2298/FIL1712875B.

[2] A. Aghajani and N. Sabzali, "Existence of coupled fixed points via measure of noncompactness and applications." Journal of nonlinear and convex analysis, vol. 14, no. 5, pp. 941-951, 2014.

[3] R. R. Akhmerov, M. I. Kamenskii, A. S. Potapov, A. E. Rodkina, and B. N. Sadovskii, Measures of Noncompactness and Condensing Operators. Basel: Birkhäuser, 1992. doi: 10.1007/978-30348-5727-7.

[4] A. Amini-Harandi, "Fixed and coupled fixed points of a new type set-valued contractive mappings in complete metric spaces," Fixed Point Theory and Applications, vol. 2012:215, pp. 1-7, 2012, doi: 10.1186/1687-1812-2012-215.

[5] J. Banaś, "Measure of noncompactness in the space of continuous tempered functions." Demonstratio Math, vol. 14, pp. 127-133, 1981. 
[6] J. Banas and K. Goebel, Measure of noncompactness in Banach spaces, Lecture Notes in Math., 60, Dekker, New York, 1980.

[7] T. G. Bhaskar and V. Lakshmikantham, "Fixed point theorems in partially ordered metric spaces and applications." Nonlinear Analysis, vol. 65, pp. 1379-1393, 2006, doi: 10.1016/j.na.2005.10.017.

[8] G. Darbo, "Punti uniti in trasformazioni a codominio non compatto," Rendiconti del Seminario Matematico della Universita di Padova, vol. 24, pp. 84-92, 1955.

[9] R. Estrada and R. P. Kanwal, Singular integral equations. Business Media New York: Birkhäuser, 200. doi: 10.1007/978-1-4612-1382-6.

[10] C. Kuratowski, "Sur les espaces complets," Fundamenta Mathematicae, vol. 15, no. 1, pp. 301309, 1930.

[11] I. K. Lifanov, L. N. Poltavskii, and G. M. Vainikko, Hypersingular Integral equations and their Applications. CRC Press, 2004.

[12] N. Muskhelishvilli, Singular Integral Equations: Boundary problems of function Theory and their Applications to Mathematical Physics. Springer Netherlands, 1958. doi: 10.1007/978-94-0099994-7.

[13] A. Samadi and M. B. Ghaemi, "An extension of darbo fixed point theorem and its applications to coupled fixed point and integral equations." Filomat, vol. 28:4, pp. 879-886, 2014, doi: 10.2298/FIL1404879S.

[14] A. Samadi and M. B. Ghaemi, "An extention of darbos theorem and its application." Abstract and Applied Analysis, vol. 2014, pp. 1-11, 2014, doi: 10.1155/2014/852324.

Author's address

A. Samadi

Department of Mathematics, Miyaneh Branch, Islamic Azad University, Miyaneh, Iran

E-mail address: samadiayub@m-iau.ac.ir 